\title{
Approximate controllability and regularity for nonlinear differential equations
}

\author{
Jin-Mun Jeong ${ }^{1 *}$, Jin-Ran $\mathrm{Kim}^{2}$ and Eun-Young Ju'
}

\author{
* Correspondence: jmjeong@pknu. \\ ac.kr \\ 'Department of Applied \\ Mathematics, Pukyong National \\ University, Busan 608-737, Korea \\ Full list of author information is \\ available at the end of the article
}

\begin{abstract}
In this article, we deal with the existence, uniqueness, and a variation of solutions of the nonlinear control system with nonlinear monotone hemicontinuous and coercive operator. Moreover, the approximate controllability for the given nonlinear control system is studied.
\end{abstract}

Keywords: nonlinear differential equation, regularity, reachable set, degree theory, approximately controllable

\section{Introduction}

Let $H$ and $V$ be two real separable Hilbert spaces such that $V$ is a dense subspace of $H$. We are interested in the following nonlinear differential control system on $H$ :

$$
\left\{\begin{array}{l}
x^{\prime}(t)+A x(t)=g\left(t, x_{t}, \int_{0}^{t} k\left(t, s, x_{s}\right) d s\right)+(B u)(t), \quad 0<t, \\
x(0)=\phi^{0}, \quad x(s)=\phi^{1}(s) \quad-h \leq s \leq 0,
\end{array}\right.
$$

where the nonlinear term, which is a Lipschitz continuous operator, is a semilinear version of the quasi-linear form. The principal operator $A$ is assumed to be a single valued, monotone operator, which is hemicontinuous and coercive from $V$ to $V^{*}$. Here, $V^{*}$ stands for the dual space of $V$. Let $U$ be a Banach space of control variables. The controller $B$ is a linear-bounded operator from a Banach space $L^{2}(0, T ; U)$ to $L^{2}(0, T ; H)$ for any $T>0$. Let the nonlinear mapping $k$ be Lipschitz continuous from $\mathbb{R} \times[-h, 0] \times V$ into $H$. If the right-hand side of the equation (SE) belongs to $L^{2}\left(0, T ; V^{*}\right)$, then it is well known as the quasi-autonomous differential equation(see Theorem 2.6 of Chapter III in [1]).

The problem of existence for solutions of semilinear evolution equations in Banach spaces has been established by several authors [1-3]. We refer to [2,4,5] to see the existence of solutions for a class of nonlinear evolution equations with monotone perturbations

First, we begin with the existence, and a variational constant formula for solutions of the equation (SE) on $L^{2}(0, T ; V) \cap W^{1,2}\left(0, T ; V^{*}\right)$, which is also applicable to optimal control problem. We prove the existence and uniqueness for solution of the equation by converting the problem into a fixed point problem. Thereafter, based on the regularity results for solutions of (SE), we intend to establish the approximate controllability for (SE). The controllability results for linear control systems have been proved by many authors, and several authors have extended these concepts to infinite dimensional semilinear system

(C) 2011 Jeong et al; licensee Springer. This is an Open Access article distributed under the terms of the Creative Commons Attribution License (http://creativecommons.org/licenses/by/2.0), which permits unrestricted use, distribution, and reproduction in any medium, provided the original work is properly cited. 
(see [6-8]). In recent years, as for the controllability for semilinear differential equations, Carrasco and Lebia [9] discussed sufficient conditions for approximate controllability of parabolic equations with delay, and Naito [10] and other authors [6-8,11] proved the approximate controllability under the range conditions of the controller $B$.

The previous results on the approximate controllability of a semilinear control system have been proved as a particular case of sufficient conditions for the approximate solvability of semilinear equations, assuming either that the semigroup generated by $A$ is a compact operator or that the corresponding linear system (SE) when $g \equiv 0$ is approximately controllable. However, Triggian [12] proved that the abstract linear system is never exactly controllable in an infinite dimensional space when the semigroup generated by $A$ is compact. Thus, we will establish the approximate controllability under more general conditions on the nonlinear term and the controller.

Our aim in this article is to establish the approximate controllability for (SE) under a stronger assumption that $\left\{y: y(t)=(B u)(t), u \in L^{2}(0, T ; U)\right\}$ is dense subspace of $L^{2}(0$, $T, H$ ), which is reasonable and widely used in case of the nonlinear system. We show that the input to solution (control to state) map is compact by using the fact that $L^{2}(0$, $T ; V) \cap W^{1,2}\left(0, T ; V^{*}\right)$ furnished with the usual topology is compactly embedded in $L^{2}(0, T, H)$, provided that the injection $V \subset H$ is compact.

In the last section, we give a simple example to which the range conditions of the controller can be applied.

\section{Nonlinear functional equations}

Let $H$ and $V$ be two real Hilbert spaces. Assume and $V$ is dense subspace in $H$ and the injection of $V$ into $H$ is continuous. If $H$ is identified with its dual space, then we may write $V \subset H \subset V^{*}$ densely, and the corresponding injections are continuous. The norm on $V$ (resp. $H$ ) will be denoted by $\|\cdot\|$ (resp. $|\cdot|)$. The duality pairing between the element $v_{1}$ of $V^{*}$ and the element $v_{2}$ of $V$ is denoted by $\left(v_{1}, v_{2}\right)$, which is the ordinary inner product in $H$ if $v_{1}, v_{2} \in H$. For the sake of simplicity, we may consider

$$
\|u\|_{*} \leq|u| \leq\|u\|, \quad u \in V
$$

where $\|\cdot\|^{*}$ is the norm of the element of $V^{*}$. If an operator $A$ is bounded linear from $V$ to $V^{*}$ and generates an analytic semigroup, then it is easily seen that

$$
H=\left\{x \in V^{*}: \int_{0}^{T}\left\|A e^{t A} x\right\|_{*}^{2} d t<\infty\right\},
$$

for the time $T>0$. Therefore, in terms of the intermediate theory we can see that

$$
\left(V, V^{*}\right)_{\frac{1}{2}, 2}=H
$$

where $\left(V, V^{*}\right)_{\frac{1}{2}, 2}$ denotes the real interpolation space between $V$ and $V^{*}$.

We note that a nonlinear operator $A$ is said to be hemicontinuous on $V$ if

$$
w-\lim _{t \rightarrow 0} A(x+t y)=A x
$$

for every $x, y \in V$ where " $w$ - lim" indicates the weak convergence on $V^{*}$. Let $A: V \rightarrow$ $V^{*}$ be given a single-valued, monotone operator and hemicontinuous from $V$ to $V^{*}$ such that 
(A1) $A(0)=0,(A u-A v, u-v) \geq \omega_{1}\|u-v\|^{2}-\omega_{2}|u-v|^{2}$,

(A2) $\|A u\|_{*} \leq \omega_{3}(\|u\|+1)$

for every $u, v \in V$ where $\omega_{2} \in \mathbb{R}$ and $\omega_{1}, \omega_{3}$ are some positive constants.

Here, we note that if $0 \neq A(0)$, then we need the following assumption:

$$
(A u, u) \geq \omega_{1}|| u \|^{2}-\omega_{2}|u|^{2}
$$

for every $u \in V$. It is also known that $A$ is maximal monotone, and $R(A)=V^{*}$ where $R(A)$ denotes the range of $A$.

Let the controller $B$ is a bounded linear operator from a Banach space $L^{2}(0, T ; U)$ to $L^{2}(0, T ; H)$ where $U$ is a Banach space.

For each $t \in[0, T]$, we define $x_{t}:[-h, 0] \rightarrow H$ as

$$
x_{t}(s)=x(t+s), \quad-h \leq s \leq 0 .
$$

We will set

$$
\Pi=L^{2}(-h, 0 ; V) \text { and } \mathbb{R}^{+}=[0, \infty) .
$$

Let $\mathcal{L}$ and $\mathscr{B}$ be the Lebesgue $\sigma$-field on $[0, \infty)$ and the Borel $\sigma$-field on $[-h, 0]$ respectively. Let $k: \mathbb{R}^{+} \times \mathbb{R}^{+} \times \Pi \rightarrow H$ be a nonlinear mapping satisfying the following:

(K1) For any $x . \in \Pi$ the mapping $k(\cdot, \cdot, x$.) is strongly $\mathcal{L} \times \mathscr{B}$-measurable;

(K2) There exist positive constants $K_{0}$, and $K_{1}$ such that

$$
\begin{aligned}
& \left|k(t, s, x .)-k\left(t, s, y_{.}\right)\right| \leq K_{1}|| x .-y . \|_{\Pi}, \\
& |k(t, s, 0)| \leq K_{0}
\end{aligned}
$$

for all $(t, s) \in \mathbb{R}^{+} \times[-h, 0]$ and $x, y, \in \Pi$.

Let $g: \mathbb{R}^{+} \times \Pi \times H \rightarrow H$ be a nonlinear mapping satisfying the following:

(G1) For any $x \in \Pi, y \in H$ the mapping $g(\cdot, x, y)$ is strongly $\mathcal{L}$-measurable;

(G2) There exist positive constants $L_{0}, L_{1}$, and $L_{2}$ such that

$$
\begin{aligned}
& \left|g(t, x ., y)-g\left(t, \hat{x}_{.}, \hat{y}\right)\right| \leq L_{1}|| x .-\hat{x} .\left|\|_{\Pi}+L_{2}\right| y-\hat{y} \mid, \\
& |g(t, 0,0)| \leq L_{0}
\end{aligned}
$$

for all $t \in \mathbb{R}^{+}, x, \hat{x} . \in \Pi$, and $y, \hat{y} \in H$.

Remark 2.1. The above operator $g$ is the semilinear case of the nonlinear part of quasi-linear equations considered by Yong and Pan [13].

For $x \in L^{2}(-h, T ; V), T>0$ we set

$$
G(t, x)=g\left(t, x_{t}, \int_{0}^{t} k\left(t, s, x_{s}\right) d s\right) .
$$

Here, as in [13], we consider the Borel measurable corrections of $x(\cdot)$.

Lemma 2.1. Let $x \in L^{2}(-h, T ; V)$. Then, the mapping $t \mapsto x_{t}$ belongs to $C([0, T] ; \Pi)$ and

$$
\|x .\|_{L^{2}(0 . T ; \Pi)} \leq \sqrt{T}\|x\|_{L^{2}(-h, T ; V)} .
$$

Proof. It is easy to verify the first paragraph and (2.1) is a consequence of the estimate:

$$
\begin{aligned}
\|x .\|_{L^{2}(0 . T ; \Pi)}^{2} & \leq \int_{0}^{T}\left\|x_{t}\right\|_{\Pi}^{2} d t \leq \int_{0}^{T} \int_{-h}^{0}\|x(t+s)\|^{2} d s d t \\
& \leq \int_{0}^{T} d t \int_{-h}^{T}\|x(s)\|^{2} d s \leq T\|x\|_{L^{2}(-h, T ; V)}^{2} .
\end{aligned}
$$


Lemma 2.2. Let $x \in L^{2}(-h, T ; V)$, $T>0$. Then, $G(\cdot, x) \in L^{2}(0, T ; H)$ and

$$
\begin{aligned}
& \|G(\cdot, x)\|_{L^{2}(0, T ; H)} \leq L_{0} \sqrt{T}+L_{2} K_{0} T^{3 / 2} / \sqrt{3} \\
& +\left(L_{1} \sqrt{T}+L_{2} K_{1} T^{3 / 2} / \sqrt{2}\right)\|x\|_{L^{2}(-h, T ; V) .}
\end{aligned}
$$

Moreover, if $x_{1}, x_{2} \in L^{2}(-h, T ; V)$, then

$$
\left\|G\left(\cdot, x_{1}\right)-G\left(\cdot, x_{2}\right)\right\|_{L^{2}(0, T ; H)} \leq\left(L_{1} \sqrt{T}+L_{2} K_{1} T^{3 / 2} / \sqrt{2}\right)\left\|x_{1}-x_{2}\right\|_{L^{2}(-h, T ; V)} .
$$

Proof. It follows from (K2) and (2.1) that

$$
\begin{aligned}
& \left\|\int_{0}^{\cdot} k\left(\cdot, s, x_{s}\right) d s\right\|_{L^{2}(0, T ; H)} \leq\left\|\int_{0}^{\cdot} k(\cdot, s, 0) d s\right\|_{L^{2}(0, T ; H)} \\
& +\left\|\int_{0}\left(k\left(\cdot, s, x_{s}\right)-k(\cdot, s, 0)\right) d s\right\|_{L^{2}(0, T ; H)} \\
& \leq K_{0} T^{3 / 2} / \sqrt{3}+\left\{\int_{0}^{T}\left|\int_{0}^{t} K_{1}\left\|x_{s}\right\|_{\Pi} d s\right|^{2} d t\right\}^{1 / 2} \\
& \leq K_{0} T^{3 / 2} / \sqrt{3}+\left\{\int_{0}^{T} K_{1}^{2} t \int_{0}^{t}\left\|x_{s}\right\|_{\Pi}^{2} d s d t\right\}^{1 / 2} \\
& \leq K_{0} T^{3 / 2} / \sqrt{3}+K_{1} T / \sqrt{2}\|x \cdot\|_{L^{2}(0, T ; \Pi)} \\
& \leq K_{0} T^{3 / 2} / \sqrt{3}+K_{1} T^{3 / 2} / \sqrt{2}\|x\|_{L^{2}(-h, T ; V)}
\end{aligned}
$$

and hence, from (G2), (2.1), and the above inequality, it is easily seen that

$$
\begin{aligned}
& \|G(\cdot, x)\|_{L^{2}(0, T ; H)} \leq\|G(\cdot, 0)\|+\|G(\cdot, x)-G(\cdot, 0)\| \\
& \leq L_{0} \sqrt{T}+L_{1}\|x \cdot\|_{L^{2}(0, T ; \Pi)}+L_{2}\left\|\int_{0} k\left(\cdot, s, x_{s}\right) d s\right\|_{L^{2}(0, T ; H)} \\
& \leq L_{0} \sqrt{T}+L_{1} \sqrt{T}\|x\|_{L^{2}(-h, T ; V)} \\
& +L_{2}\left(K_{0} T^{3 / 2} / \sqrt{3}+K_{1} T^{3 / 2} / \sqrt{2}\|x\|_{L^{2}(-h, T ; V)}\right) .
\end{aligned}
$$

Similarly, we can prove (2.3).

Let us consider the quasi-autonomous differential equation

$$
\left\{\begin{array}{l}
x^{\prime}(t)+A x(t)=f(t), \quad 0<t \leq T \\
x(0)=\phi^{0}
\end{array}\right.
$$

where $A$ satisfies the hypotheses mentioned above. The following result is from Theorem 2.6 of Chapter III in [1].

Proposition 2.1. Let $\Phi^{0} \in H$ and $f \in L^{2}\left(0, T ; V^{*}\right)$. Then, there exists a unique solution $x$ of $(E)$ belonging to

$$
C([0, T] ; H) \cap L^{2}(0, T ; V) \cap W^{1,2}\left(0, T ; V^{*}\right)
$$

and satisfying

$$
\begin{aligned}
& |x(t)|^{2}+\int_{0}^{t}\|x(s)\|^{2} d s \leq C_{1}\left(\left|\phi^{0}\right|^{2}+\int_{0}^{t}\|f(s)\|_{*}^{2} d s\right), \\
& \int_{0}^{t}\left\|\frac{d x(s)}{d s}\right\|_{*}^{2} d t \leq C_{1}\left(\left|\phi^{0}\right|^{2}+\int_{0}^{t}\|f(s)\|_{*}^{2} d s\right)
\end{aligned}
$$

where $C_{1}$ is a constant. 
Acting on both sides of $(\mathrm{E})$ by $x(t)$, we have

$$
\frac{1}{2} \frac{d}{d t}|x(t)|^{2}+\left.\omega_{1}|| x(t)\right|^{2} \leq \omega_{2}|x(t)|^{2}+(f(t), x(t)) .
$$

As is seen Theorem 2.6 in [1], integrating from 0 to $t$, we can determine the constant $C_{1}$ in Proposition 2.1.

We establish the following result on the solvability of the equation (SE).

Theorem 2.1. Let $A$ and the nonlinear mapping $g$ be given satisfying the assumptions mentioned above. Then, for any $\left(\Phi^{0}, \Phi^{1}\right) \in H \times L^{2}(-h, 0 ; V)$ and $f \in L^{2}\left(0, T ; V^{*}\right), T$ $>0$, the following nonlinear equation

$$
\left\{\begin{array}{l}
x^{\prime}(t)+A x(t)=G(t, x)+f(t), \quad 0<t \leq T \\
x(0)=\phi^{0}, \quad x(s)=\phi^{1}(s), \quad-h \leq s \leq 0
\end{array}\right.
$$

has a unique solution $x$ belonging to

$$
L^{2}(-h, T ; V) \cap W^{1,2}\left(0, T ; V^{*}\right) \subset C([0, T] ; H)
$$

and satisfying that there exists a constant $C_{2}$ such that

$$
\|x\|_{L^{2}(-h, T ; V) \cap W^{1,2}(0, T ; V *)} \leq C_{2}\left(1+\left|\phi^{0}\right|+\left\|\phi^{1}\right\|_{L^{2}(-h, 0 ; V)}+\|f\|_{L^{2}(0, T ; V *)}\right) .
$$

Proof. Let $y \in L^{2}(0, T ; V)$. Then, we extend it to the interval $(-h, 0)$ by setting $y(s)=$ $\Phi^{1}(s)$ for $s \in(-h, 0)$, and hence, $G(\cdot, y(\cdot)) \in L^{2}(0, T ; H)$ from Lemma 2.2. Thus, by virtue of Proposition 2.1, we know that the problem

$$
\left\{\begin{array}{l}
x^{\prime}(t)+A x(t)=G(t, y)+f(t), \quad 0<t \\
x(0)=\phi^{0}, \quad x(s)=\phi^{1}(s) \quad-h \leq s \leq 0
\end{array}\right.
$$

has a unique solution $x_{y} \in L^{2}(0, T ; V) \cap W^{1,2}\left(0, T ; V^{*}\right)$ corresponding to $y$. Let us fix $T_{0}>0$ so that

$$
\omega_{1}^{-1} e^{\omega_{2} T_{0}}\left(L_{1} \sqrt{T}_{0}+L_{2} K_{1} T_{0}^{3 / 2} / \sqrt{2}\right)<1
$$

Let $x_{i}, i=1,2$, be the solution of (2.8) corresponding to $y_{i}$. Multiplying by $x_{1}(t)-x_{2}$ $(t)$, we have that

$$
\begin{aligned}
& \left(\dot{x}_{1}(t)-\dot{x}_{2}(t), x_{1}(t)-x_{2}(t)\right)+\left(A x_{1}(t)-A x_{2}(t), x_{1}(t)-x_{2}(t)\right) \\
& =\left(G\left(t, y_{1}\right)-G\left(t, y_{2}\right), x_{1}(t)-x_{2}(t)\right),
\end{aligned}
$$

and hence it follows that

$$
\begin{aligned}
& \frac{1}{2} \frac{d}{d t}\left|x_{1}(t)-x_{2}(t)\right|^{2}+\omega_{1}|| x_{1}(t)-x_{2}(t) \|^{2} \\
& \left.\leq \omega_{2}\left|x_{1}(t)-x_{2}(t)\right|^{2}+\| G\left(t, y_{1}\right)\right)-G\left(t, y_{2}\right)\|\|_{*}|| x_{1}(t)-x_{2}(t) \| .
\end{aligned}
$$

From Lemma 2.2 and integrating over $[0, t]$, it follows

$$
\begin{aligned}
& \frac{1}{2}\left|x_{1}(t)-x_{2}(t)\right|^{2}+\omega_{1} \int_{0}^{t}\left\|x_{1}(s)-x_{2}(s)\right\|^{2} d s \\
& \leq \frac{1}{2 c} \int_{0}^{t}\left\|G\left(s, y_{1}\right)-G\left(s, y_{2}\right)\right\|_{*}^{2} d s \\
& +\frac{c}{2} \int_{0}^{t}\left\|x_{1}(s)-x_{2}(s)\right\|^{2} d s+\omega_{2} \int_{0}^{t}\left|x_{1}(s) d s-x_{2}(s) d s\right|^{2} d s,
\end{aligned}
$$


where $c$ is a positive constant satisfying $2 \omega_{1}-c>0$. Here, we used that

$$
a b \leq \frac{a^{p}}{p}+\frac{b^{q}}{q}, \quad p^{-1}+q^{-1}=1(1<p<\infty)
$$

for any pair of nonnegative numbers $a$ and $b$. Thus, from (2.3) it follows that

$$
\begin{aligned}
& \left|x_{1}(t)-x_{2}(t)\right|^{2}+\left(2 \omega_{1}-c\right) \int_{0}^{t} \| x_{1}(s) d s-\left.x_{2}(s) d s\right|^{2} d s \\
& \leq c^{-1}\left(L_{1} \sqrt{T}_{0}+L_{2} K_{1} T_{0}^{3 / 2} / \sqrt{2}\right)^{2} \int_{0}^{t}\left\|y_{1}(s)-y_{2}(s)\right\|^{2} d s \\
& +2 \omega_{2} \int_{0}^{t}\left|x_{1}(s)-x_{2}(s)\right|^{2} d s .
\end{aligned}
$$

By using Gronwall's inequality, we get

$$
\begin{aligned}
& \left|x_{1}\left(T_{0}\right)-x_{2}\left(T_{0}\right)\right|^{2}+\left(2 \omega_{1}-c\right) \int_{0}^{T_{0}}\left\|x_{1}(s)-x_{2}(s)\right\|^{2} d s \\
& \leq c^{-1}\left(L_{1} \sqrt{T}_{0}+L_{2} K_{1} T_{0}^{3 / 2} / \sqrt{2}\right)^{2} e^{2 \omega_{2} T_{0}} \int_{0}^{T_{0}}\left\|y_{1}(s)-y_{2}(s)\right\|^{2} d s .
\end{aligned}
$$

Taking $c=\omega_{1}$, it holds that

$$
\begin{aligned}
\left\|x_{1}-x_{2}\right\|_{L^{2}\left(0, T_{0} ; V\right)} & \leq \omega_{1}^{-1} e^{\omega_{2} T_{0}}\left(L_{1} \sqrt{T}_{0}\right. \\
& \left.+L_{2} K_{1} T_{0}^{3 / 2} / \sqrt{2}\right)\left\|y_{1}-y_{2}\right\|_{L^{2}\left(0, T_{0} ; V\right)} .
\end{aligned}
$$

Hence, we have proved that $y \mapsto x$ is a strictly contraction from $L^{2}\left(0, T_{0} ; V\right)$ to itself if the condition (2.9) is satisfied. It shows that the equation (2.6) has a unique solution in $\left[0, T_{0}\right]$.

From now on, we derive the norm estimates of solution of the equation (2.6). Let $y$ be the solution of

$$
\left\{\begin{array}{l}
y^{\prime}(t)+A y(t)=f(t), \quad 0<t \leq T_{0} \\
y(0)=\phi^{0}
\end{array}\right.
$$

Then,

$$
\frac{d}{d t}(x(t)-\gamma(t))+(A x(t)-A y(t))=G(t, x),
$$

by multiplying by $x(t)-y(t)$ and using the assumption (A1), we obtain

$$
\begin{aligned}
& \frac{1}{2} \frac{d}{d t}|x(t)-\gamma(t)|^{2}+\omega_{1}\|x(t)-\gamma(t)\|^{2} \\
& \leq \omega_{2}|x(t)-\gamma(t)|^{2}+\|G(t, x)\|_{*}\|x(t)-\gamma(t)\| .
\end{aligned}
$$

By integrating over $[0, t]$ and using Gronwall's inequality, we have

$$
\begin{aligned}
& \|x-y\|_{L^{2}\left(0, T_{0} ; V\right)} \leq \omega_{1}^{-1} e^{\omega_{2} T_{0}}\|G(\cdot, x)\|_{L^{2}\left(0, T_{0} ; V *\right)} \\
& \leq \omega_{1}^{-1} e^{\omega_{2} T_{0}}\left\{L_{0} \sqrt{T_{0}}+L_{2} K_{0} T_{0}^{3 / 2} / \sqrt{3}\right. \\
& +\left(L_{1} \sqrt{T}_{0}+L_{2} K_{1} T_{0}^{3 / 2} / \sqrt{2}\right)\left(\|x\|_{L^{2}\left(0, T_{0} ; V\right)}+\left\|\phi^{1}\right\|_{\left.L^{2}(-h, 0 ; V)\right\},}\right.
\end{aligned}
$$


and hence, putting

$$
N=\omega_{1}^{-1} e^{\omega_{2} T_{0}} \text { and } L=L_{1} \sqrt{T}_{0}+L_{2} K_{1} T_{0}^{3 / 2} / \sqrt{2}
$$

it holds

$$
\begin{aligned}
& \|x\|_{L^{2}\left(0, T_{0} ; V\right)} \leq \frac{N}{1-N L}\left(L_{0} \sqrt{T}_{0}+L_{2} K_{0} T_{0}^{3 / 2} / \sqrt{3}\right) \\
& \left.+\frac{1}{1-N L}\|y\|_{L^{2}\left(0, T_{0} ; V\right)}+\frac{N L}{1-N L}\left\|\phi^{1}\right\|_{L^{2}(-h, 0 ; V}\right) \\
& \leq \frac{N}{1-N L}\left(L_{0} \sqrt{T}_{0}+L_{2} K_{0} T_{0}^{3 / 2} / \sqrt{3}\right) \\
& +\frac{C_{1}}{1-N L}\left(\left|\phi^{0}\right|+\|f\|_{L^{2}\left(0, T_{0} ; V *\right)}\right) \\
& +\frac{N L}{1-N L}\left\|\phi^{1}\right\|_{L^{2}(-h, 0 ; V)} \\
& \leq C_{2}\left(1+\left|\phi^{0}\right|+\left\|\phi^{1}\right\|_{L^{2}(-h, 0 ; V)}+\|f\|_{L^{2}\left(0, T_{0} ; V *\right)}\right)
\end{aligned}
$$

for some positive constant $C_{2}$. Since the condition (2.9) is independent of initial values, the solution of (2.6) can be extended to the internal $\left[0, n T_{0}\right]$ for natural number $n$, i.e., for the initial value $\left(x\left(n T_{0}\right), x_{n} T_{0}\right)$ in the interval $\left[n T_{0},(n+1) T_{0}\right]$, as analogous estimate (2.11) holds for the solution in $\left[0,(n+1) T_{0}\right]$.

Theorem 2.2. If $\left.\left(\Phi^{0}, \Phi^{1}\right) \in H \times L^{2}(-h, 0, V)\right)$ and $f \in L^{2}\left(0, T ; V^{*}\right)$, then $x \in L^{2}(-h$, $T ; V)) \cap W^{1,2}\left(0, T ; V^{*}\right)$, and the mapping

$$
\left.H \times L^{2}(-h, 0 ; V) \times L^{2}\left(0, T ; V^{*}\right) \ni\left(\phi^{0}, \phi^{1}, f\right) \mapsto x \in L^{2}(-h, T ; V)\right) \cap W^{1,2}\left(0, T ; V^{*}\right)
$$

is continuous.

Proof. It is easy to show that if $\left.\left(\Phi^{0}, \Phi^{1}\right) \in H \times L^{2}(-h, 0 ; V)\right)$ and $f \in L^{2}\left(0, T ; V^{*}\right)$ for every $T>0$, then $x$ belongs to $L^{2}(-h, T ; V) \cap W^{1,2}\left(0, T ; V^{*}\right)$. Let

$$
\left(\phi_{i}^{0}, \phi_{i}^{1}, f_{i}\right) \in H \times L^{2}(-h, 0 ; V) \times L^{2}\left(0, T_{1} ; V^{*}\right)
$$

and $x_{i}$ be the solution of (2.6) with $\left(\phi_{i}^{0}, \phi_{i}^{1}, f_{i}\right)$ in place of $\left(\phi^{0}, \phi^{1}, f\right)$ for $i=1,2$.

Then, in view of Proposition 2.1 and Lemma 2.2, we have

$$
\begin{aligned}
& \frac{1}{2} \frac{d}{d t}\left|x_{1}(t)-x_{2}(t)\right|^{2}+\omega_{1}|| x_{1}(t)-x_{2}(t) \|^{2} \\
& \leq \omega_{2}\left|x_{1}(t)-x_{2}(t)\right|^{2}+\left\|G\left(t, x_{1}\right)-G\left(t, x_{2}\right)\right\|_{*}\left\|x_{1}(t)-x_{2}(t)\right\| \\
& +\left\|f_{1}(t)-f_{2}(t)\right\|_{*}|| x_{1}(t)-x_{2}(t) \|
\end{aligned}
$$

If $\omega_{1}-c / 2>0$, we can choose a constant $c_{1}>0$ so that

$$
\omega_{1}-c / 2-c_{1} / 2>0
$$

and

$$
\begin{aligned}
\left.\| f_{1}(t)-f_{2}(t)\right)\|\|_{*}\left\|x_{1}(t)-x_{2}(t)\right\| & \leq \frac{1}{2 c_{1}}\left\|f_{1}(t)-f_{2}(t)\right\|_{*}^{2} \\
& +\frac{c_{1}}{2}\left\|x_{1}(t)-x_{2}(t)\right\|^{2} .
\end{aligned}
$$

Let $T_{1}<T$ be such that

$$
2 \omega_{1}-c-c_{1}-c^{-1} e^{2 \omega_{2} T_{1}}\left(L_{1} \sqrt{T_{1}}+L_{2} K_{1} T_{1}^{3 / 2} / \sqrt{2}\right)^{2}>0 .
$$


Integrating on (2.12) over $\left[0, T_{1}\right]$ and as is seen in the first part of proof, it follows

$$
\begin{aligned}
& \left(2 \omega_{1}-c-c_{1}\right)\left\|x_{1}-x_{2}\right\|_{L^{2}\left(0, T_{1} ; V\right)}^{2} \leq e^{2 \omega_{2} T_{1}}\left\{\left|\varphi_{1}^{0}-\varphi_{2}^{0}\right|^{2}\right. \\
& \left.+\frac{1}{c}\left\|G\left(t, x_{1}\right)-G\left(t, x_{2}\right)\right\|_{L^{2}\left(0, T_{1} ; V *\right)}^{2}+\frac{1}{c_{1}}\left\|f_{1}-f_{2}\right\|_{L^{2}\left(0, T_{1} ; V *\right)}^{2}\right\} \\
& \leq e^{2 \omega_{2} T_{1}}\left\{\left|\phi_{1}^{0}-\phi_{2}^{0}\right|^{2}\right. \\
& +\frac{1}{c}\left(L_{1} \sqrt{T_{1}}+L_{2} K_{1} T_{1}^{3 / 2} / \sqrt{2}\right)^{2}|| x_{1}-x_{2} \|_{L^{2}\left(-h, T_{1} ; V\right)}^{2} \\
& \left.+\frac{1}{c_{1}}\left\|f_{1}-f_{2}\right\|_{L^{2}\left(0, T_{1} ; V *\right)}^{2}\right\} .
\end{aligned}
$$

Putting that

$$
N_{1}=2 \omega_{1}-c-c_{1}-c^{-1} e^{2 \omega_{2} T_{1}}\left(L_{1} \sqrt{T_{1}}+L_{2} K_{1} T_{1}^{3 / 2} / \sqrt{2}\right)^{2}
$$

we have

$$
\begin{gathered}
\left\|x_{1}-x_{2}\right\|_{L^{2}\left(0, T_{1} ; V\right)} \leq \frac{e^{\omega_{2} T_{1}}}{N_{1}^{1 / 2}}\left(\left|\phi_{1}^{0}-\phi_{2}^{0}\right|+\frac{1}{c_{1}}\left\|f_{1}-f_{2}\right\|_{L^{2}\left(0, T_{1} ; V *\right)}\right) \\
+\frac{c^{-1 / 2} e^{\omega_{2} T_{1}}\left(L_{1} \sqrt{T_{1}}+L_{2} K_{1} T_{1}^{3 / 2} / \sqrt{2}\right)}{N_{1}^{1 / 2}}\left\|\phi_{1}^{1}-\phi_{2}^{1}\right\|_{L^{2}(-h, 0 ; V) .} .
\end{gathered}
$$

Suppose that

$$
\left.\left(\phi_{n}^{0}, \phi_{n}^{1}, f_{n}\right) \rightarrow\left(\phi^{0}, \phi^{1}, f\right) \text { in } H \times L^{2}(-h, 0 ; V)\right) \times L^{2}\left(0, T ; V^{*}\right),
$$

and let $x_{n}$ and $x$ be the solution (2.6) with $\left(\phi_{n}^{0}, \phi_{n}^{1}, f_{n}\right)$ and $\left(\phi^{0}, \phi^{1}, f\right)$ respectively.

By virtue of (2.13) with $T$ being replaced by $T_{1}$, we see that

$$
\left.x_{n} \rightarrow x \quad \text { in } \quad L^{2}\left(-h, T_{1} ; V\right)\right) \cap W^{1,2}\left(0, T_{1} ; V^{*}\right) \subset C\left(\left[0, T_{1}\right] ; H\right) .
$$

This implies that $\left(x_{n}\left(T_{1}\right),\left(x_{n}\right)_{T_{1}}\right) \rightarrow\left(x\left(T_{1}\right), x_{T_{1}}\right)$ in $H \times L^{2}(-h, 0 ; V)$. Hence, the same argument shows that

$$
x_{n} \rightarrow x \quad \text { in } L^{2}\left(T_{1}, \min \left\{2 T_{1}, T\right\} ; V\right) \cap W^{1,2}\left(T_{1}, \min \left\{2 T_{1}, T\right\} ; V^{*}\right) .
$$

Repeating this process, we conclude that

$$
x_{n} \rightarrow x \quad \text { in } \quad L^{2}(-h, T ; V) \cap W^{1,2}\left(0, T ; V^{*}\right) .
$$

Remark 2.2. For $x \in L^{2}(0, T ; V)$, we set

$$
G(t, x)=\int_{0}^{t} k(t-s) g(s, x(s)) d s
$$

where $k$ belongs to $L^{2}(0, T)$ and $g:[0, T] \times V \rightarrow H$ be a nonlinear mapping satisfying

$$
|g(t, x)-g(t, y)| \leq L|| x-y||
$$

for a positive constant L. Let $x L L^{2}(0, T ; V), T>0$. Then, $G(\cdot, x) L L^{2}(0, T ; H)$ and

$$
\|G(\cdot, x)\|_{L^{2}(0, T ; H)} \leq L|| k\left\|_{L^{2}(0, T)} \sqrt{T}\right\| x \|_{L^{2}(0, T ; V)} .
$$

Moreover, if $x_{1}, x_{2} L L^{2}(0, T ; V)$, then

$$
\left\|G\left(\cdot, x_{1}\right)-G\left(\cdot, x_{2}\right)\right\|_{L^{2}(0, T ; H)} \leq L\|k\| \sqrt{T}\left\|x_{1}-x_{2}\right\|_{L^{2}(0, T ; V)} .
$$


Then, with the condition that

$$
\omega_{1}^{-1} e^{\omega_{2} T_{0}} L\|k\| \sqrt{T_{0}}<1
$$

in place of the condition (2.9), we can obtain the results of Theorem 2.1.

\section{Approximate controllability}

In what follows we assume that the embedding $V \subset H$ is compact, and $A$ is a continuous operator from $V$ to $V^{*}$ satisfying (A1) and (A2). For $h L L^{2}(0, T ; H)$ and let $x_{h}$ be the solution of the following equation with $B=I$ :

$$
\left\{\begin{array}{l}
x^{\prime}(t)+A x(t)=G(t, x)+h(t), \quad 0<t \\
x(0)=0 \quad x(s)=0 \quad-h \leq s \leq 0
\end{array}\right.
$$

where

$$
G(t, x)=g\left(t, x_{t}, \int_{0}^{t} k\left(t, s, x_{s}\right) d s\right)
$$

We define the solution mapping $S$ from $L^{2}\left(0, T ; V^{*}\right)$ to $L^{2}(0, T ; V)$ by

$$
(S h)(t)=x_{h}(t), h \in L^{2}\left(0, T ; V^{*}\right) .
$$

Let $\mathcal{A}$ and $\mathcal{G}$ be the Nemitsky operators corresponding to the maps $A$ and $G$, which are defined by $\mathcal{A}(x)(\cdot)=A x(\cdot)$ and $\mathcal{G}(h)(\cdot)=G\left(\cdot, x_{h}\right)$, respectively. Then, since the solution $x$ belongs to $L^{2}(-h, T ; V) \cap W^{1,2}\left(0, T ; V^{*}\right) \subset C([0, T] ; H)$, it is represented by

$$
x_{h}(t)=\int_{0}^{t}((I+\mathcal{G}-\mathcal{A} S) h)(s) d s,
$$

and with aid of Lemma 2.2 and Proposition 2.1

$$
\begin{aligned}
& \|S h\|_{L^{2}(0, T ; V) \cap W^{1,2}\left(0, T ; V^{*}\right)}=\left\|x_{h}\right\| \leq C_{1}\left\|G\left(\cdot, x_{h}\right)+h\right\|_{L^{2}(0, T ; V *)} \\
& \leq C_{1}\left\{L_{0} \sqrt{T}+L_{2} K_{0} T^{3 / 2} / \sqrt{3}+\left(L_{1} \sqrt{T}+L_{2} K_{1} T^{3 / 2} / \sqrt{2}\right)\|x\|_{L^{2}(0, T ; V)}\right. \\
& \left.+\|h\|_{L^{2}(0, T ; V *)}\right\} \\
& \leq C_{1}\left\{L_{0} \sqrt{T}+L_{2} K_{0} T^{3 / 2} / \sqrt{3}\right. \\
& \left.+\left(L_{1} \sqrt{T}+L_{2} K_{1} T^{3 / 2} / \sqrt{2}\right)\left(1+\|h\|_{L^{2}(0, T ; V *)}\right)+\|h\|_{L^{2}(0, T ; V *)}\right\} .
\end{aligned}
$$

Hence, if $h$ is bounded in $L^{2}\left(0, T ; V^{*}\right)$, then so is $x_{h}$ in $L^{2}(0, T ; V) \cap W^{1,2}\left(0, T ; V^{*}\right)$. Since $V$ is compactly embedded in $H$ by assumption, the embedding $L^{2}(0, T ; V) \cap W^{1,2}$ $\left(0, T ; V^{*}\right) \subset L^{2}(0, T ; H)$ is compact in view of Theorem 2 of Aubin [14]. Hence, the mapping $h \mapsto S h=x_{h}$ is compact from $\mathrm{L}^{2}\left(0, T ; V^{*}\right)$ to $L^{2}(0, T ; H)$. Therefore, $\mathcal{G}$ is a compact mapping from $L^{2}\left(0, T ; V^{*}\right)$ to $L^{2}(0, T ; H)$ and so is $\mathcal{A} S$ from $L^{2}\left(0, T ; V^{*}\right)$ to itself. The solution of (SE) is denoted by $x(T ; g, u)$ associated with the nonlinear term $g$ and control $u$ at time $T$.

Definition 3.1. The system (SE) is said to be approximately controllable at time $T$ if $C l\left\{x(T ; g, u): u \in L^{2}(0, T ; U)\right\}=V^{*}$ where $C l$ denotes the closure in $V^{*}$.

We assume

(T) $1-\omega_{1}^{-1} \omega_{3} e^{\omega_{2} T}>0$

(B) $C l\left\{y: y(t)=(B u)(t)\right.$, a.e. $\left.\left.u \in L^{2}(0, T ; U)\right\}=L^{2}(0, T ; U)\right\}$. Here $C l$ is the closure in $L^{2}(0, T ; H)$. 
Theorem 3.1. Let the assumptions (T) and (B) be satisfied. Then,

$$
C l\left\{(I-\mathcal{A} S) h: h \in L^{2}\left(0, T ; V^{*}\right)\right\}=L^{2}\left(0, T ; V^{*}\right) .
$$

Therefore, the following nonlinear differential control system

$$
\left\{\begin{array}{l}
\frac{d x(t)}{d t}+A x(t)=(B u)(t), \quad 0<t \leq T, \\
x(0)=x_{0}
\end{array}\right.
$$

is approximately controllable at time $T$.

Proof. Let $z L L^{2}\left(0, T ; V^{*}\right)$ and $r$ be a constant such that

$$
z \in U_{r}=\left\{x \in L^{2}\left(0, T ; V^{*}\right):\|x\|_{L^{2}\left(0, T ; V^{*}\right)}<r\right\} .
$$

Take a constant $d>0$ such that

$$
\left(r+\omega_{3}+N_{2}\left|x_{0}\right|\right)\left(1-N_{2}\right)^{-1}<d,
$$

where

$$
N_{2}=\omega_{1}^{-1} \omega_{3} e^{\omega_{2} T} .
$$

Taking scalar product on both sides of (3.1) with $G=0$ by $x(t)$

$$
\begin{aligned}
\frac{1}{2} \frac{d}{d t}|x(t)|^{2} & +\omega_{1}\|x(t)\|^{2} \leq \omega_{2}|x(t)|^{2}+\|h(t)\|\left\|_{*}\right\| x(t) \| \\
& \leq \omega_{2}|x(t)|^{2}+\frac{1}{2 c}\|h(t)\|_{*}^{2}+\frac{c}{2}\|x(t)\|^{2}
\end{aligned}
$$

where $c$ is a positive constant satisfying $2 \omega_{1}-c>0$. Integrating on $[0, t]$, we get

$$
\begin{aligned}
\frac{1}{2}|x(t)|^{2}+\omega_{1} \int_{0}^{t}\|x(s)\|^{2} d s & \leq \frac{1}{2}\left|x_{0}\right|^{2}+\frac{1}{2 c} \int_{0}^{t}\|h(s)\|_{*}^{2} d s \\
& +\frac{c}{2} \int_{0}^{t}\|x(s)\|^{2} d s+\omega_{2} \int_{0}^{t}|x(s)|^{2} d s,
\end{aligned}
$$

and hence,

$$
\begin{aligned}
|x(t)|^{2}+\left(2 \omega_{1}-c\right) \int_{0}^{t}\|x(s)\|^{2} d s & \leq\left|x_{0}\right|^{2}+\frac{1}{c} \int_{0}^{t}\|h(s)\|_{*}^{2} d s \\
& +2 \omega_{2} \int_{0}^{t}|x(s)|^{2} d s .
\end{aligned}
$$

By using Gronwall's inequality, it follows that

$$
|x(T)|^{2}+\left(2 \omega_{1}-c\right) \int_{0}^{T}\|x(s)\|^{2} d s \leq e^{2 \omega_{2} T}\left(\left|x_{0}\right|^{2}+\frac{1}{c} \int_{0}^{T}\|h(s)\|_{*}^{2} d s\right),
$$

that is,

$$
\begin{aligned}
\|S h\|_{L^{2}(0, T ; V)} & =\|x\|_{L^{2}(0, T ; V)} \\
\leq & e^{\omega_{2} T}\left(2 \omega_{1}-c\right)^{-1 / 2}\left(\left|x_{0}\right|+c^{-1 / 2}\|h\|_{L^{2}\left(0, T ; V^{*}\right)}\right) .
\end{aligned}
$$

Let us consider the equation

$$
z=(I-\mathcal{A} S) w .
$$


Let $w$ be the solution of (3.9). Then $z L U_{d}$ and taking $c=\omega_{1}$, from (3.7), (3.8)

$$
\begin{aligned}
\|w\|_{L^{2}\left(0, T ; V^{*}\right)} & \leq\|z\|_{L^{2}\left(0, T ; V^{*}\right)}+\|\mathcal{A S} w\|_{L^{2}\left(0, T ; V^{*}\right)} \\
\leq & r+\omega_{3}\left(\|S w\|_{L^{2}\left(0, T ; V^{*}\right)}+1\right) \\
\leq & r+\omega_{3}\left\{\omega_{1}^{-1 / 2} e^{\omega_{2} T}\left(\left|x_{0}\right|+\omega_{1}^{-1 / 2}\|w\|\right)+1\right\}
\end{aligned}
$$

and hence

$$
\|w\| \leq\left(r+\omega_{3}+N_{2}\left|x_{0}\right|\right)\left(1-N_{2}\right)^{-1}<d
$$

it follows that $w \notin \partial U_{d}$ where $\partial U_{d}$ stands for the boundary of $U_{d}$. Thus, the homotopy property of topological degree theory there exists $w L L^{2}\left(0, T ; V^{*}\right)$ such that the equation (3.9) holds. Based on the assumption (B), there exists a sequence $\left\{u_{n}\right\} L L^{2}(0$, $T$; $U$ ) such that $B u_{n} \mapsto w$ in $L^{2}\left(0, T ; V^{*}\right)$. Then, by the last paragraph of Theorem 2.1, we have that $x\left(\cdot ; g, u_{n}\right) \mapsto x_{w}$ in $L^{2}(0, T ; V) \cap W^{1,2}\left(0, T ; V^{*}\right) \subset C([0, T] ; H)$. Hence, we have proved (3.5). Let $y L V^{*}$. Then, there exists an element $u L L^{2}(0, T ; U)$ such that

$$
\left\|\frac{y}{T}-(I-\mathcal{A} S) B u\right\|_{L^{2}\left(0, T ; V^{*}\right)}<\frac{\varepsilon}{\sqrt{T}} .
$$

Thus

$$
\begin{aligned}
\|y-x(T)\|_{*} & =\left\|y-\int_{0}^{T}((I-\mathcal{A} S) B u)(s) d s\right\|_{*} \\
& \leq \int_{0}^{T}\left\|\frac{y}{T}-((I-\mathcal{A} S) B u)(s)\right\|_{*} d s \\
& \leq \sqrt{T}\left\|\frac{y}{T}-(I-\mathcal{A} S) B u\right\|_{L^{2}(0, T ; V *)}<\varepsilon .
\end{aligned}
$$

Therefore, the system (3.6) is approximately controllable at time $T$.

In order to investigate the controllability of the nonlinear control system, we need to impose the following condition.

(F) $g$ is uniformly bounded: there exists a constant $M_{g}$ such that

$$
|g(t, x, y)| \leq M_{g}
$$

for all $x, y L V$.

By (F) it holds that

$$
\|G(\cdot, x)\|_{L^{2}(0, T ; H)} \leq M_{g} \sqrt{T},
$$

and for every $h L L^{2}\left(0, T ; V^{*}\right)$

$$
\|\mathcal{G}(h)\|_{L^{2}(0, T ; H)} \leq M_{g} \sqrt{T}
$$

Theorem 3.2. Let the assumptions (T), (B), and (F) be satisfied. Then, we have

$$
C l\left\{(\mathcal{G}+I-\mathcal{A S}) h: h \in L^{2}\left(0, T ; V^{*}\right)\right\}=L^{2}\left(0, T ; V^{*}\right) .
$$

Thus, the system (SE) is approximately controllable at time $T$.

Proof. Let $U_{r}$ be the ball with radius $r$ in $L^{2}\left(0, T ; V^{*}\right)$ and $z L U_{r}$. To prove (3.11), we will also use the degree theory for the equation

$$
z=\lambda(\mathcal{G}-\mathcal{A S}) w+w, \quad 0 \leq \lambda \leq 1
$$


in open ball $U_{d}$ where the constant $d$ satisfies

$$
\left(r+\omega_{3}+N_{2}\left|x_{0}\right|+M_{g} \sqrt{T}\right)\left(1-N_{2}\right)^{-1}<d
$$

where the constant $N_{2}$ is in Theorem 3.1. If $w$ is the solution of (3.12), then $z L U_{d}$ and from Lemma 2.1

$$
\begin{aligned}
& \|w\|_{L^{2}(0, T ; V *)} \leq\|z\|+\|\mathcal{A S} w\|+\|\mathcal{G} w\| \\
& \left.\quad \leq r+\omega_{3}(\|S w\|+1)+M_{g} \sqrt{T}\right) \\
& \quad \leq r+\omega_{3}\left\{\omega_{1}^{-1 / 2} e^{\omega_{2} T}\left(\left|x_{0}\right|+\omega_{1}^{-1 / 2}|| w \|\right)+1\right\}+M_{g} \sqrt{T},
\end{aligned}
$$

and hence

$$
\|w\| \leq\left(r+\omega_{3}+N_{2}\left|x_{0}\right|+M_{g} \sqrt{T}\right)\left(1-N_{2}\right)^{-1}<d
$$

it follows that $w \notin \partial U_{d}$. Hence, there exists $w L L^{2}\left(0, T ; V^{*}\right)$ such that the equation (3.12) holds. Using the similar way to the last part of Theorem 3.1 and the assumption (B), there exists a sequence $\{u n\} L L^{2}(0, T ; U)$ such that $B u_{n} \mapsto w$ in $L^{2}\left(0, T ; V^{*}\right)$ and $x$ $\left(\cdot, g, u_{n}\right) \mapsto x_{w}$ in $L^{2}(0, T ; V) \cap W^{1,2}\left(0, T ; V^{*}\right) \subset C([0, T] ; H)$. Thus, we have proved (3.11), and the system (1.1) is approximately controllable at time $T$.

\section{Example}

Let $-A$ be an operator associated with a bounded sesquilinear form $a(u, v)$ defined in $V$ $\times V$ and satisfying Gårding inequality

$$
\operatorname{Re} a(u, v) \geq c_{0}|| u \|^{2}-c_{1}|u|^{2}, \quad c_{0}>0, \quad c_{1} \geq 0
$$

for any $u L V$. It is known that $A$ generates an analytic semigroup in both $H$ and $V^{*}$. By virtue of the Riesz-Schauder theorem, if the embedding $V \subset H$ is compact, then the operator $A$ has discrete spectrum:

$$
\sigma(A)=\left\{\mu_{n}: n=1,2, \ldots\right\}
$$

which has no point of accumulation except possibly when $\mu=\infty$. Let $\mu_{n}$ be a pole of the resolvent of $A$ of order $k_{n}$ and $P_{n}$ the spectral projection associated with $\mu_{n}$

$$
P_{n}=\frac{1}{2 \pi i} \int_{\Gamma_{n}}(\mu-A)^{-1} d \mu,
$$

where $\Gamma_{n}$ is a small circle centered at $\mu_{n}$ such that it surrounds no point of $\sigma(A)$ except $\mu_{n}$. Then, the generalized eigenspace corresponding to $\mu_{n}$ is given by

$$
H_{n}=P_{n} H=\left\{P_{n} u: u \in H\right\},
$$

and we have that from $P_{n}^{2}=P_{n}$ and $H_{n} \subset V$; it follows that

$$
P_{n} V=\left\{P_{n} u: u \in V\right\}=H_{n} .
$$

Definition 4.1. The system of the generalized eigenspaces of $A$ is complete in $\mathrm{H}$ if $\mathrm{Cl}$ $\left\{\operatorname{span}\left\{H_{n}: n=1,2, \ldots\right\}\right\}=H$ where $\mathrm{Cl}$ denotes the closure in $H$.

We need the following hypotheses:

(B1) The system of the generalized eigenspaces of $A$ is complete. 
(B2) There exists a constant $d>0$ such that

$$
\|v\| \leq d\|B v\|, \quad v \in L^{2}(0, T ; U) .
$$

We can see many examples which satisfy (B2)(cf. $[8,11])$.

Consider about the intercept controller $B$ define $\mathrm{d}$ by

$$
(B u)(t)=\sum_{n=1}^{\infty} u_{n}(t)
$$

where

$$
u_{n}=\left\{\begin{array}{cc}
0, & 0 \leq t \leq \frac{T}{n} \\
P_{n} u(t), & \frac{T}{n}<t \leq T .
\end{array}\right.
$$

Hence, we see that $u_{1}(t) \equiv 0$ and $u_{n}(t) L \operatorname{Im} P_{n}$.

First of all, for the meaning of the condition (B) in section 3, we need to show the existence of controller satisfying $\mathrm{Cl}\left\{B u: u L L^{2}(0, T ; U)\right\} \neq L^{2}(0, T ; H)$. In fact, by completion of the generalized eigenspaces of $A$, we may write that $f(t)=\sum_{n=1}^{\infty} P_{n} f(t)$ for $\in L^{2}(0, T ; H)$. Let us choose $f \in L^{2}(0, T ; H)$ satisfying

$$
\int_{0}^{T}\left\|P_{1} f(t)\right\|^{2} d t>0
$$

Then, since

$$
\begin{aligned}
& \int_{0}^{T}\|f(t)-B u(t)\|^{2} d t=\int_{0}^{T} \sum_{n=1}^{\infty}\left\|P_{n}(f(t)-B u(t))\right\|^{2} d t \\
& \geq \int_{0}^{T}\left\|P_{1}(f(t)-B u(t))\right\|^{2} d t=\int_{0}^{T}\left\|P_{1} f(t)\right\|^{2} d t>0,
\end{aligned}
$$

the statement mentioned above is reasonable.

Let $f \in L^{2}(0, T ; H)$ and $\alpha=T /(T-T / n)$. Then we know

$$
f(\cdot) \equiv \alpha K_{[T, T / n]} f(\alpha(\cdot-T / n)) \text { in } L^{2}(0, T ; H),
$$

where $K_{[T, T / n]}$ is the characteristic of $[T, T / n]$. Define

$$
w(s)=\sum_{n=1}^{\infty} w_{n}(s), \quad w_{n}(s)=\alpha K_{[T, T / n]} B^{-1} P_{n} f(\alpha(s-T / n)) .
$$

Thus $(B w)(t)=\sum_{n=1}^{\infty} P_{n} f(s)$, a.e. Since the system of the generalized eigenspaces of $A$ is complete, it holds that for every $f L L^{2}(0, T ; H)$ and $\in>0$

$$
\left\|f(\cdot)-\sum_{n=1}^{\infty} P_{n} f(\cdot)\right\|_{L^{2}(0, T ; H)}=\|f(\cdot)-B w\|_{L^{2}(0, T ; H)}<\varepsilon
$$

Thus, the intercept controller $B$ define d by (4.1) satisfies the condition (B). 


\section{Author details}

'Department of Applied Mathematics, Pukyong National University, Busan 608-737, Korea ${ }^{2}$ Department of Mathematics, Dong-A University Saha-Gu, Busan 604-714, Korea

\section{Authors' contributions}

JMJ carried out the main proof of this manuscript, JRK drafted the manuscript and corrected the main theorems, EYJ conceived of the study, and participated in its design and coordination.

\section{Competing interests}

The authors declare that they have no competing interests.

Received: 24 March 2011 Accepted: 23 August 2011 Published: 23 August 2011

\section{References}

1. Barbu, V: Nonlinear Semigroups and Differential Equations in Banach space. Noordho Leiden, Netherland (1976)

2. Ahmed, NU, Xiang, X: Existence of solutions for a class of nonlinear evolution equations with nonmonotone perturbations. Nonlinear Anal Theory Methods Appl. 22(1), 81-89 (1994). doi:10.1016/0362-546X(94)90007-8

3. Pazy, A: Semigroup of Linear Operator and Applications to Partial Differential Equations. Springer-Verlag, New York (1983)

4. Aizicovici, S, Papageorgiou, NS: Infinite dimensional parametric optimal control problems. Jpn J Ind Appl Math. 10, 307-332 (1993). doi:10.1007/BF03167579

5. Hirano, N: Nonlinear evolution equations with nonmonotonic perturbations. Nonlinear Anal Theory Methods Appl. 13(6), 599-609 (1989). doi:10.1016/0362-546X(89)90081-3

6. Jeong, JM, Ju, JE, Lee, KY: Controllability for variational inequalities of parabolic type with nonlinear pertirbation. J Inequal Appl 2010, 1-16 (2010). Article ID 768469

7. Sukavanam, N, Tomar, Nutan Kumar: Approximate controllability of semilinear delay control system. Nonlinear Func Anal Appl. 12, 53-59 (2007)

8. Zhou, HX: Approximate controllability for a class of semilinear abstract equations. SIAM J Control Optim. 21, 551-565 (1983). doi:10.1137/0321033

9. Carrasco, A, Lebia, H: Approximate controllability of a system of parabolic equations with delay. J Math Anal Appl. 345 , 845-853 (2008). doi:10.1016/j.jmaa.2008.04.068

10. Naito, K: Controllability of semilinear control systems dominated by the linear part. SIAM J Control Optim. 25, 715-722 (1987). doi:10.1137/0325040

11. Zhou, HX: Controllability properties of linear and semilinear abstract control systems. SIAM J Control Optim. 22, 405-422 (1984). doi:10.1137/0322026

12. Triggiani, R: A note on the lack of exact controllability for mild solutions in Banach spaces. SIAM J Control Optim. 15 407-411 (1977). doi:10.1137/0315028

13. Yong, J, Pan, L: Quasi-linear parabolic partial differential equations with delays in the highest order partial derivatives. J Aust Math Soc. 54, 174-203 (1993). doi:10.1017/S1446788700037101

14. Aubin, JP: Un thèoréme de compacité. C R Acad Sci. 256, 5042-5044 (1963)

doi:10.1186/1687-1847-2011-27

Cite this article as: Jeong et al:: Approximate controllability and regularity for nonlinear differential equations. Advances in Difference Equations 2011 2011:27.

\section{Submit your manuscript to a SpringerOpen ${ }^{\circ}$ journal and benefit from:}

- Convenient online submission

Rigorous peer review

- Immediate publication on acceptance

- Open access: articles freely available online

- High visibility within the field

- Retaining the copyright to your article

Submit your next manuscript at $\mathbf{s p r i n g e r o p e n . c o m ~}$ 